\title{
Análise do consumo de sacarose na dieta dos pacientes em tratamento na Clínica da Faculdade de Odontologia da Ufrgs
}

\author{
Analysis of sucrose intake in the diet of patients treated at the \\ Orthodontic Clinic of the School of Dentistry, Ufrgs
}

Juliana Figueiredo dal Gallo Faria*
Márcia Cançado Figueiredo ${ }^{* *}$
Bianca de Simoni Simões
Karina Santos Mundstock

\section{Resumo}

Objetivo: verificar a frequência e a ocasião da ingestão de sacarose na dieta de pacientes atendidos na disciplina de Ortodontia Clínica da Faculdade de Odontologia da Universidade Federal do Rio Grande do Sul. Sujeitos e método: o presente estudo analisou, por intermédio do índice de potencial cariogênico e da solicitação de diário alimentar de três dias, a ingestão de sacarose na dieta de pacientes. Além disso, o índice do potencial cariogênico foi associado ao sexo e à idade dos pacientes. Resultados: do total de 82 questionários enviados, houve retorno de 44 (53,7\%). A média total do potencial cariogênico foi de 69 pontos $( \pm 24,5)$, o que corresponde a um escore moderado. Entretanto, 18 pacientes (40,9\%) tiveram escore alto do potencial cariogênico, 22 pacientes (50\%) escore médio e, somente, 4 pacientes $(9,1 \%)$ apresentaram escore baixo. $O$ índice do potencial cariogênico não apresentou associação significativa nem com o sexo $(p=0,727)$ nem com a faixa etária $(p=0,742)$ dos pacientes. Houve associação estatística significativa da média do consumo de sacarose entre as refeições com o escore do potencial cariogênico $\left(r_{s}=0,616 ; p<0,001\right)$. Conclusão: a maior parte dos pacientes $(90,9 \%)$ tem uma dieta com escore de moderado a alto em relação ao potencial cariogênico. Não foi encontrada associação estatisticamente significativa do potencial cariogênico com o sexo e a faixa etária dos pacientes.

Palavras-chave: Cárie dentária. Dieta. Ortodontia.

\section{Introdução}

Uma das doenças crônicas mais comuns da infância é a cárie dentária, por isso, é considerada um problema de saúde pública. No entanto, deve-se considerar que essa é uma doença que pode ser prevenida e controlada ${ }^{1}$.

Os açúcares presentes na dieta (sacarose, glicose, frutose e lactose) são utilizados pelas bactérias do biofilme para seu metabolismo energético ${ }^{2}$. Assim, a atividade metabólica das bactérias, por meio da produção de ácidos orgânicos, causa o desenvolvimento da doença cárie devido à desmineralização de tecidos dentários pela baixa do $\mathrm{pH}$. Os ácidos (láctico, acético, propiônico e fórmico) atuam sobre os íons de cálcio e fosfato da superfície dos cristais de hidroxiapatita, presentes nos tecidos mineralizados, causando a difusão desses para fora do dente e, consequentemente, a desmineralização desse ${ }^{3,4}$.

Dessa forma, a dieta assume um importante papel na etiologia da doença cárie ${ }^{5}$, já que fornece os substratos, influencia na produção de ácidos, no tipo e na quantidade de biofilme bacteriano, na composição de microrganismos e na qualidade e quantidade de secreção salivar. A maioria dos estudos sobre dieta e cárie demonstra a relação de ingestão de alimentos ricos em açúcar (um tipo de carboidrato) com o aparecimento da cárie, independente da idade ${ }^{6}$.

Graduada em Odontologia pela Universidade Federal do Rio Grande do Sul (Ufrgs), Porto Alegre, Rio Grande do Sul, Brasil.

Doutora em Odontopediatria. Professora Associada IV do Departamento de Odontopediatria da FO-Ufrgs, Porto Alegre, Rio Grande do Sul, Brasil.

** Graduada em Odontologia pela Ufrgs, Porto Alegre, Rio Grande do Sul, Brasil.

${ }^{* * *}$ Doutora em Ortodontia. Professora Adjunta III do Departamento de Ortodontia da FO-Ufrgs, Porto Alegre, Rio Grande do Sul, Brasil. 
Os conhecimentos atuais direcionam para a aplicação de uma nova filosofia para o tratamento da cárie dentária, que se contrapõe com os antigos procedimentos adotados, que focavam apenas no tratamento da sequela da doença, ou seja, suas cavidades evidentes. Atualmente, o procedimento cirúrgico restaurador deve ser visto como parte do tratamento, inserido em um conjunto de medidas capazes de realmente combater a doença. Entre essas medidas estão: motivação do paciente para escovações regulares dos dentes, controle da dieta, utilização do flúor e consultas frequentes ao cirurgião-dentista, procedimentos que devem ser intensificados para evitar o aparecimento da cárie ${ }^{7}$.

Geralmente, quando inseridos em um tratamento ortodôntico, os pacientes encontram-se cárie-inativos. Entretanto, ser considerado paciente cárie-inativo não significa que a sua dieta não seja rica em sacarose e que não contribua para o surgimento de futuras lesões. Principalmente pelo fato de que pacientes que estão sob tratamento ortodôntico, seja em tratamento com aparelhos móveis ou fixos, têm uma higiene bucal mais difícil de ser realizada, o que os tornam, consequentemente, mais propícios ao desenvolvimento da doença cárie ${ }^{8}$. Dessa forma, esses pacientes precisam controlar os outros fatores que influenciam o surgimento da doença cárie, como $o$ alto índice de ingestão de sacarose.

Assim, o objetivo deste estudo foi analisar a frequência e a ocasião do consumo de sacarose na dieta de pacientes em atendimento na disciplina de Ortodontia Clínica da Faculdade de Odontologia da Universidade Federal do Rio Grande do Sul (FO-Ufrgs).

\section{Sujeitos e método}

Este projeto de pesquisa foi analisado e aprovado pelos comitês de pesquisa e ética da Faculdade de Odontologia da Universidade Federal do Rio Grande do Sul sob o número 24279.

A metodologia utilizada foi a abordagem exploratória e descritiva, utilizando o método quantitativo por meio do preenchimento de ficha clínica e de diário alimentar. O público-alvo foi um grupo de pacientes, com idades de 4 a 12 anos, atendidos durante o segundo semestre de 2013 e o ano de 2014 na Clínica de Ortodontia da graduação da FO-Ufrgs.

Foram selecionados pacientes que estavam em tratamento com aparelhos removíveis, uma vez que o tipo de aparelho pode influenciar a dieta e a higienização dos dentes. Desse modo, optou-se por analisar esse grupo porque a maioria dos pacientes da clínica estava realizando esse tipo de tratamento, que modifica menos os hábitos alimentares dos pacientes, uma vez que o aparelho é removido durante a alimentação ${ }^{9}$.

No primeiro momento, os pais ou responsáveis foram esclarecidos sobre a pesquisa e lhes foi solicitada autorização para participação dos pacientes, a qual foi efetivada por meio do preenchimento do Termo de Consentimento Livre e Esclarecido. Em um segundo momento, foi solicitado o preenchimento de um diário alimentar de três dias, no qual o responsável foi orientado a descrever tudo o que o paciente comesse e bebesse nas principais refeições e também entre elas, colocando os horários e a quantidade dessas.

Antes da consulta, os pesquisadores entraram em contato com os pais e/ou responsáveis por telefone para lembrá-los de trazer o diário alimentar preenchido. Dessa forma, na consulta, quando os pais e/ou responsáveis traziam o diário alimentar preenchido, os pesquisadores o revisavam e os questionavam caso faltasse algum dado. Por último, os pesquisadores fizeram rápida análise desses diários e realizaram aconselhamento dietético com os pais e/ou responsáveis e com a criança, de acordo com o padrão de refeição apresentado no diário alimentar.

Os diários alimentares foram estudados considerando o índice de potencial cariogênico de cada dieta, conforme descrito por Giancarman et al. ${ }^{10}$ (2012). Foram classificados os tipos de carboidratos ingeridos, para cada carboidrato foi atribuído um valor arbitrário baseado em suas propriedades cariogênicas. Desse modo, alimentos não pegajosos tinham numeração menor que os carboidratos açucarados e pegajosos. Também foi analisada a frequência e a ocasião da ingestão desses alimentos.

Os escores de frequência e ocasião foram multiplicados pelos valores de cada tipo de alimento e os números resultantes equivalem ao índice de frequência (IF) e ao índice de ocasião (IO). O potencial cariogênico (PC) da dieta de cada paciente foi obtido pela soma do IF e do IO. Assim, os valores obtidos a partir de cada variável foram convertidos para uma escala de três categorias, como descrito na Tabela 1.

Tabela 1 - Classificação do potencial cariogênico

\begin{tabular}{l|c}
\hline \multicolumn{1}{c|}{ Potencial cariogênico } & \multicolumn{2}{c}{ Escore } \\
\hline Baixo & $10-33$ \\
Moderado & $34-79$ \\
Alto & $80-144$ \\
\hline
\end{tabular}

Fonte: Giancarman et al. ${ }^{10}(2012)$.

As variáveis quantitativas foram descritas pela média e pelo desvio padrão e as categóricas por meio das frequências absolutas e relativas. Para avaliar a associação entre as variáveis categóricas, foi aplicado o teste Qui-Quadrado de Pearson. Na associação entre as variáveis quantitativas, os testes da correlação de Pearson (distribuição simétrica) ou Spearman (distribuição assimétrica) foram aplicados. O nível de significância adotado foi de 5\% ( $p \leq 0,05)$ e as análises foram realizadas no programa SPSS versão 21.0. 


\section{Resulltados}

Do total de 82 questionários enviados, houve retorno de 44 instrumentos (53,7\%). Dessa forma, neste estudo, foi utilizado um nível de confiança de $95 \%$ e uma margem de erro de $15 \%$, considerando-se o seu caráter predominantemente descritivo, sendo a margem de erro considerada mais relevante do que seu poder de erro ${ }^{11,12}$.

A média de idade dos participantes deste estudo foi de 10,5 anos $( \pm 2,4)$, havendo predominância do sexo feminino $(\mathrm{n}=26 ; 59,1 \%)$. A média do escore total do potencial cariogênico foi de 69 pontos $( \pm 24,5)$. A classificação dos pacientes conforme PC está apresentada na Tabela 2.

Tabela 2 - Distribuição dos pacientes quanto ao potencial cariogênico

\begin{tabular}{l|r}
\hline \multicolumn{1}{c|}{ Potencial cariogênico } & $\mathrm{n}(\%)$ \\
\hline Baixo $(10-33)$ & $4(9,1)$ \\
Moderado $(34-79)$ & $22(50,0)$ \\
Alto $(80-144)$ & $18(40,9)$
\end{tabular}

Fonte: dados da pesquisa.

A média do consumo de sacarose entre as refeições foi de $2,5( \pm 1,0)$. Somente 2 pacientes $(4,5 \%)$ consumiam 5 ou mais refeições com sacarose entre as refeições principais. Não houve associação estatisticamente significativa entre a média do consumo de sacarose nas refeições principais com o escore do PC ( $r=-0,148 ; p=0,349)$. No entanto, houve associação estatisticamente significativa da média do consumo de sacarose entre as refeições com o escore do PC $\left(r_{s}=0,616 ; p<0,001\right)$. Pacientes com maior consumo de sacarose entre as refeições tiveram um escore mais elevado de PC, conforme apresenta a Figura 1.

Figura 1 - Associação entre consumo de sacarose entre as refeições com o escore $P C$

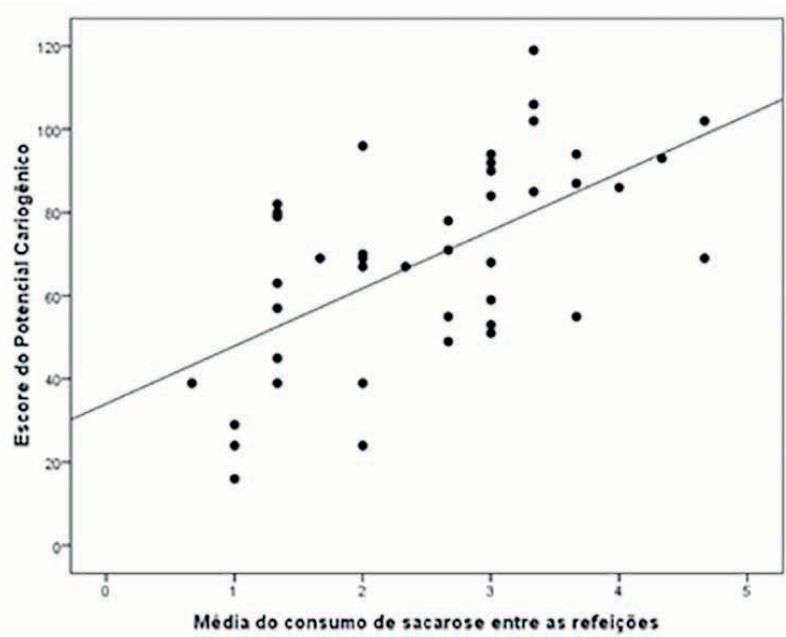

Fonte: dados da pesquisa
Não houve associação estatisticamente significativa entre o potencial cariogênico e o sexo do paciente $(\mathrm{p}=0,727)$, como mostra a Tabela 3.

Tabela 3 - Classificação do potencial cariogênico conforme sexo

\begin{tabular}{l|r|r|c}
\hline Potencial cariogênico & \multicolumn{1}{|c|}{$\begin{array}{c}\text { Masculino } \\
\mathrm{n}(\%)\end{array}$} & \multicolumn{1}{|c|}{$\begin{array}{c}\text { Feminino } \\
\mathrm{n}(\%)\end{array}$} & $\mathrm{p}$ \\
\hline Baixo $(10-33)$ & $1(5,6)$ & $3(11,5)$ & \\
Moderado $(34-79)$ & $10(55,6)$ & $12(46,2)$ & 0,727 \\
Alto $(80-144)$ & $7(38,9)$ & $11(42,3)$ & \\
\hline
\end{tabular}

Também não houve associação estatisticamente significativa entre o potencial cariogênico com a faixa etária do paciente $(p=0,742)$, como apresenta a Tabela 4. Os pacientes foram classificados em menores ou iguais a 10 anos ou maiores de 10 anos de idade, pelo fato de que a média de idade do estudo foi de 10,5 anos $( \pm 2,4)$.

Tabela 4 - Classificação do potencial cariogênico conforme faixa etária

\begin{tabular}{l|r|r|c}
\hline \multicolumn{1}{c|}{$\begin{array}{c}\text { Potencial } \\
\text { cariogênico }\end{array}$} & $\begin{array}{c}\leq 10 \text { anos } \\
(\mathrm{n}=25 ; 56,8 \%) \\
\mathrm{n}(\%)\end{array}$ & $\begin{array}{c}>10 \text { anos } \\
(\mathrm{n}=19 ; 43,2 \%) \\
\mathrm{n}(\%)\end{array}$ & $\mathrm{p}$ \\
\hline $\begin{array}{l}\text { Baixo } \\
(10-33)\end{array}$ & $3(12,0)$ & $1(5,3)$ & \\
$\begin{array}{l}\text { Moderado } \\
(34-79)\end{array}$ & $12(48,0)$ & $10(52,6)$ & 0,742 \\
$\begin{array}{l}\text { Alto } \\
(80-144)\end{array}$ & $10(40,0)$ & $8(42,1)$ & \\
\hline
\end{tabular}

Fonte: dados da pesquisa.

A Tabela 5 mostra a classificação do potencial cariogênico conforme a faixa etária dos pacientes, mostrando que há uma predominância de pacientes entre 10-12 anos tanto para o sexo masculino quanto para o feminino. Ainda, observa-se que também não houve associação significativa entre as variáveis $(r=0,016 ; p=0,918)$ quando considerado o escore de forma contínua com a idade. 
Tabela 5 - Classificação do sexo e do potencial cariogênico conforme faixa etária

\begin{tabular}{|c|c|c|c|c|c|}
\hline & \\
\hline & 4-6 anos & 7-9 anos & 10-12 anos & 13-15 anos & $16-20$ anos \\
\hline $\begin{array}{l}\text { Masculino } \\
\qquad(\mathrm{n}=18 ; 40,9 \%)\end{array}$ & $1(5,55 \%)$ & $5(27,75 \%)$ & $9(49,95 \%)$ & $2(11,1 \%)$ & $1(5,55 \%)$ \\
\hline $\begin{array}{l}\text { Feminino } \\
\qquad(\mathrm{n}=26 ; 59,1 \%)\end{array}$ & $1(3,85 \%)$ & $7(26,95 \%)$ & $13(50.05 \%)$ & $5(19,25 \%)$ & $0(0 \%)$ \\
\hline $\begin{array}{l}\text { Total } \\
\qquad(\mathrm{n}=44 ; 100 \%)\end{array}$ & $2(4,54 \%)$ & $12(27,24 \%)$ & $22(49,94 \%)$ & $7(15,89 \%)$ & $1(2,27 \%)$ \\
\hline $\begin{array}{l}\text { PC baixo } \\
\qquad(\mathrm{n}=4 ; 9,1 \%)\end{array}$ & $0(0 \%)$ & $2(50 \%)$ & $2(50 \%)$ & $0(0 \%)$ & $0(0 \%)$ \\
\hline $\begin{array}{l}\text { PC moderado } \\
\qquad(\mathrm{n}=22 ; 50 \%)\end{array}$ & $0(0 \%)$ & $6(27 \%)$ & $10(45 \%)$ & $5(22,5 \%)$ & $1(4,5 \%)$ \\
\hline $\begin{array}{l}\text { PC alto } \\
\qquad(\mathrm{n}=18 ; 40,9 \%)\end{array}$ & $2(11,1 \%)$ & $4(22,2 \%)$ & $10(55,5 \%)$ & $2(11,1 \%)$ & $0(0 \%)$ \\
\hline
\end{tabular}

Fonte: dados da pesquisa.

\section{Discussão}

Há uma grande evidência epidemiológica da influência da dieta cariogênica no aparecimento e desenvolvimento da doença cárie ${ }^{13,14}$. Desse modo, a dieta pode ser analisada por meio de questionários $^{15-18}$ ou por intermédio da solicitação de diários alimentare $\mathrm{s}^{10,19,20}$.

No presente estudo, a dieta foi analisada por meio de um diário alimentar de três dias, cuja taxa de retorno foi de $53,7 \%$. Os pacientes frequentavam a clínica de ortodontia periodicamente e, desse modo, os responsáveis eram lembrados por telefone para que levassem o diário alimentar preenchido na consulta. No entanto, esses relatavam esquecer-se de fazer o diário alimentar durante a semana, ou que não tinham como fazê-lo já que a criança também se alimentava na creche e não teria como saber o que ela ingeria nesse período. Apesar de a taxa de retorno ter sido somente um pouco acima dos $50 \%$, essa é a média encontrada em outros estudos que também utilizaram diário alimentar como forma de avaliação ${ }^{20}$.

Após a análise dos diários alimentares, a média do potencial cariogênico encontrada nesta pesquisa foi de 69 pontos $( \pm 24,5)$, o que corresponde a um escore moderado. No estudo de Giancarman et al. ${ }^{10}$ (2012), o escore médio do PC encontrado também foi moderado - 64,03 $( \pm 25,10)$. Além disso, em ambos os estudos, houve uma predominância do escore moderado, seguido pelo escore alto e, por último, o escore baixo, apesar de o presente estudo analisar somente pacientes ortodônticos. No estudo de Giancarman et al., não foi encontrado nenhum escore baixo, já no presente estudo, quatro pacientes $(9,1 \%)$ apresentaram escore baixo do PC.

Apesar de os pacientes analisados serem cárie-inativos, 90,9\% apresentaram escore moderado ou alto do PC. Esse dado pode ser explicado pelo fato de que a doença cárie é considerada uma doença infecciosa, multifatorial e transmissível, sendo resultado da combinação de três fatores principais: hospedeiro, microbiota cariogênica (Streptococcus mutans) e substrato (sacarose) ${ }^{21-23}$. A confluência desses fatores determina o desenvolvimento da doença, enquanto a ausência de um dos fatores determina a não ocorrên$\mathrm{cia}^{21}$.

Além disso, não houve associação significativa entre o PC e o sexo dos pacientes $(p=0,727)$, diferentemente do encontrado em Giancarman et al. ${ }^{10}$, que apontaram diferença significativa de PC entre homens e mulheres, com predomínio de um PC maior no sexo feminino. No presente estudo, a população era jovem, variando de 4 a 20 anos de idade, provavelmente por isso não houve diferença significativa. Já no referido estudo, a amostra variava de 12 a 67 anos, sendo que $70,90 \%$ do total de pacientes $(n=275)$ tinham mais de 20 anos, caracterizando uma população mais madura.

Neste estudo, também foi analisada a frequência da ingestão de sacarose entre as refeições e observou-se que a média desse consumo foi de 2,5 $( \pm 1,0)$. No estudo de Cangussu e Costa $^{24}$, foram entrevistados escolares na faixa estaria de 12 a 20 anos, e a média de ingestão de lanches com alto consumo de sacarose intercalado entre as refeições também foi de 2,5. Acredita-se que esse comportamento é atribuído ao baixo custo desse grupo de alimentos bem como à sua grande valorização na cultura e na dieta ocidental.

No entanto, a alta frequência de ingestão de sacarose é um alerta de risco para o desenvolvimento da doença cárie. Assim, é muito importante conscientizar os responsáveis sobre o controle dessa dieta como forma educativa. Apesar de ser muito difícil alterar hábitos dietéticos, é dever do cirurgião-dentista motivar seus pacientes, orientando para substituir alimentos cariogênicos por alimentos não cariogênicos, diminuir a frequência da ingestão de sacarose, principalmente entre as refeições, bem como melhorar a qualidade nutricional dos alimentos ingeridos. Essas orientações podem ajudar a 
prevenir o estabelecimento de novas lesões de cáries ou evitar a ativação de lesões inativas.

Novais et al. ${ }^{25}$ (2004) mediram o grau de preferência por açúcar de crianças na faixa etária dos 0 aos 12 anos por meio de soluções com diferentes concentrações de sacarose. Nos pacientes livres de cárie, não foi encontrada diferença significativa entre a preferência por açúcar (escolha entre as soluções) e entre a idade e o sexo do paciente. Dessa forma, essa pesquisa corrobora com o resultado do presente estudo, que também não encontrou associação significativa entre o consumo médio de sacarose entre as refeições principais com a idade e o sexo do paciente.

Segundo estudos clássicos realizados em humanos $^{3-26}$, a frequência de ingestão de açúcar está diretamente relacionada com o desenvolvimento de lesões de cárie. No presente estudo, houve associação significativa entre a média do consumo de sacarose entre as refeições e o escore do PC. Dessa forma, pacientes com maior consumo de sacarose entre as refeições tiveram um escore mais elevado do PC. Esses resultados concordam com o relato de Gustaffson et al. ${ }^{3}$ (1954) de que a incidência de cárie é ainda maior quando o açúcar é consumido entre as refeições. Assim, é natural haver uma associação significativa entre o potencial cariogênico de uma dieta e a média do consumo de sacarose entre as refeições.

Apesar de ter-se optado por utilizar uma metodologia nova e ainda pouco utilizada nos estudos em odontologia, o índice de potencial cariogênico permite analisar os diários alimentares quantificando a frequência e a ocasião de ingestão e o tipo de sacarose ingerida, e não apenas contar o número de vezes que o indivíduo consome sacarose, como classicamente se tem relatos na literatura. Com esta análise, classifica-se o $\mathrm{PC}$ em relação à quantidade de sacarose ingerida, se essa é ingerida nas refeições principais ou entre essas refeições e, ainda, se esse alimento é ingerido em forma líquida ou pastosa.

Estudo anterior ${ }^{3}$ conclui que alimentos ingeridos entre as refeições apresentam maior relação com a doença cárie do que alimentos ingeridos em refeições principais e, ainda, que alimentos pegajosos têm maior potencial cariogênico do que sacarose em forma líquida. Dessa forma, o método escolhido para classificar os diários alimentares em relação ao seu potencial cariogênico abrange todas as variáveis relacionadas a esse índice.

Muitas vezes, o consumo de açúcares passa despercebido pelo paciente e/ou responsável. Uma vez que eles estão presentes na maior parte dos produtos industrializados, como achocolatados, refrigerantes, biscoitos e cereais, que são frequentemente ingeridos pelas crianças. $\mathrm{O}$ aumento da ingestão desse tipo de alimento está cada vez mais frequente devido ao seu baixo custo, à praticidade, à rapidez, à durabilidade e à boa aceitação desses produtos $^{27-29}$. Além de propiciar o desenvolvimento da doença cárie, o consumo exagerado de açúcares pode trazer muitos danos à saúde dessas crianças, como obesidade ${ }^{30-32}$, diabetes ${ }^{31-33}$ e câncer ${ }^{31,32}$.

Em suma, pacientes que estão sob tratamento ortodôntico são mais propícios ao desenvolvimento da doença cárie ${ }^{8}$, uma vez que a higiene bucal se torna mais complexa com a implementação dos aparelhos ortodônticos, sejam eles móveis ou fixos. Assim, esta pesquisa proporciona dados para a programação e o desenvolvimento de uma nova ficha clínica para a área de ortodontia da FO-Ufrgs abordando o tema dieta, bem como fornece subsídios para justificar que ações preventivas na referida clínica sejam realizadas.

\section{Conclusões}

Diante dos resultados obtidos neste estudo, é lícito concluir que a maior parte dos pacientes $(90,9 \%)$ que frequentam a Clínica de Ortodontia da FO-Ufrgs tem uma dieta com escore de moderado a alto com relação ao potencial cariogênico. O consumo de sacarose entre as refeições desses sujeitos foi moderado, apresentando uma associação significativa com o escore do potencial cariogênico. Dessa forma, pacientes que estão em tratamento ortodôntico podem ser considerados mais suscetíveis ao desenvolvimento da doença cárie.

\section{Abstract}

Objective: To verify the frequency and time of sucrose intake in the diet of patients treated at the Orthodontic Clinic, School of Dentistry of the Federal University of Rio Grande do Sul (FO-Ufrgs). Subjects and method: The present study analyzed - by requesting a food intake diary for 3 (three) days - the sucrose intake in the diet of patients through the Cariogenic Potential (C.P.) index. In addition, the Cariogenic Potential index was associated with gender and age of patients. Results: From a total of 82 questionnaires sent, 44 of them were returned $(53.7 \%)$. The average of the total score of Cariogenic Potential was 69 points ( \pm 24.5$)$, corresponding to a moderate score of Cariogenic Potential. However, 18 patients (40.9\%) showed high C.P. score, 22 patients (50\%) showed an average score, and only 4 patients (9.1\%) presented a low score. There was no statistically significant association of the Cariogenic Potential index with neither gender $(p=0.727)$ nor age $(p=0.742)$ of patients. There was a significant statistical association of average sucrose intake in-between meals with $C$. $P$. score $(r=0.616 ; p<0.001)$. Conclusion: The diet of most patients $(90.9 \%)$ present moderate to high score in relation to the Cariogenic Potential index. There was no statistical significant association of the Cariogenic Potential index with gender or age of patients.

Keywords: Dental Caries. Diet. Orthodontics. 


\section{Referências}

1. Misra S, Tahmassebi JF, Brosnan M. Early childhood caries: a review. Dent Update 2007; 34(9):556-64.

2. Leites ACBR, Pinto MB, Souza ERS. Aspectos microbiológicos da cárie dental. Salusvita 2006; 25(2):135-48.

3. Gustaffson BE, Quensel CEL, Lanke LS. The effect of different levels of carbohydrate intake on caries activity in 436 individuals observed for five years. Acta Odontol Scand 1954; 11(3-4):232-364.

4. Stephan RM. Changes in hydrogenion concentration on tooth surfaces and in caries lesions. J Am Dental Associat 1940; 27:718-23.

5. Lima JO. Cárie dentária: um novo conceito. Rev Dental Press Ortodon Ortoped Facial 2007; 12(6):119-30.

6. Woodward M, Walker AR. Sugar consumption and dental caries: evidence from 90 countries. Br Dent J 1994; 176:297-302.

7. Mota LQ, Leite JSM, Targino AGR. Minimally Invasive Dentistry through the Partial Removal of Carious Dentin in Deep Cavities. Cient Ciênc Biol Saúde 2013; 15(2):145-52.

8. Olympio KPK, Bardal PAP, Henriques JFC, Bastos JRM. Prevenção de cárie dentária e doença periodontal em ortodontia: uma necessidade imprescindível. Rev Dent Press Ortodon Facial 2006; 11(2):110-19.

9. Hebling SRF, Pereira AC, Hbling E, Meneghim MC. Considerações para elaboração de protocolo de assistência ortodôntica em saúde coletiva. Cien Saúde Colet 2007; 2(4):1067-78.

10. Giancarman RA, Fernandéz CE, Díaz N. Fermentable carbohydrate dietary consumption measured by a cariogenicity scoring system and caries experience in youth and adults. Rev Chil Nutr 2012; 39(4):116-22.

11. Sidia MC. Bioestatística: princípios e aplicações. 2. ed. Porto Alegre: Artmed; 2003.

12. Stephen B, Steven R, Warren S. Delineando a pesquisa clínica: uma abordagem epidemiológica. 2. ed. Porto Alegre: Artmed; 2003.

13. Santos MN, Santos LM, Francisco SB, Cury JA. Relationship among dental plaque composition, daily sugar exposure and caries in the primary dentition. Caries Res 2002; 37(3):347-52.

14. Tinanoff N, Kanellis MJ, Vargas CM. Current understanding of the epidemiology, mechanisms and prevention of dental caries in preschool children. Pediatr Dent 2002; 24(6):543-51.

15. Peres KGA, Bastos JRM, La Torre MRDO. Severidade de cárie em crianças e relação com aspectos sociais e comportamentais. Rev Saúde Pública 2000; 34(4):402-8.

16. Bonotto DMV, Pintarelli TP, Santin G, Mante GR, Ferreira FM, Fraiz FC. Cárie dentária e gênero em adolescentes. RFO UPF 2015; 20(2):202-7.

17. ElSalhy M, Honkala S, Söderling E, Varghese A, Honkala E. Relationship between daily habits, Streptococcus mutans, and caries among schoolboys. J Dentistry 2013; 41:1000-6.

18. Moura SMS, Oliveira IM, Leite CMC, Júnior AMC. Diet and dental caries in schoolchildren from 10 to 14 years old in the city of Picos, Piauí. J Health Sci 2016; 18(1):14-8.

19. Fonseca YPC, Guedes-Pinto AC. Controle da dieta alimentar em pacientes de odontopediatria com alta incidência de cárie. Rev Assoc Paul Cir Dent 1984; 38(4):289-301.

20. Marques AP, Messer LB. Nutrient intake and dental caries in the primary dentition. Pediatr Dent 1992; 14(5):314-21.

21. Keyes PH. Recent advances in dental research. Bacteriology Int Dent J 1962; 12(4):443-64.
22. Keys PH. Present and future measures for dental caries control. J Am Dent Assoc 1969; 79(6):1395-404.

23. Ferjeskov O. Changing paradigms in concepts on dental caries: consequences for oral health care. Caries Res 2004; 38:182-91.

24. Cangussu MCT, Costa MCN. Topical fluoride in the decline in the prevalence of caries in adolescents from Salvador-BA, 1996. Pesqui Odontol Bras 2001; 15(4):348-53.

25. Novais SMA, Batalha RP, Grinfeld S, Fortes TM, Pereira MAS. Relação doença cárie - açúcar: prevalência em crianças. Pesq Bras Odontoped Clin Integr 2004; 4(3):199-203.

26. Von Der Fehr FR, Löe H, Theilade E. Experimental caries in man. Caries Res 1970; 4(2):131-48.

27. Kinsey JD. Food and families socioeconomic status. J Nutr $1994 ; 124: 1878$ S-85.

28. Waitzberg DL, Simopoulos AP, Bourne PG, Faergeman O. Obstáculos para a implementação governamental de dietas saudáveis com base científica e como superá-los. Est Avan 2013; 27(78):133-52.

29. Júnior JALAL, Gonçalves LV, Correia AA. Alimentos x cárie: a ingestão do açúcar em excesso como fator estimulante do desenvolvimento da doença. Cien Biol Saud 2015; 2(2):11-20.

30. Dias CB, Coppi A. Consumo alimentar e prevalência de sobrepeso/obesidade em pré-escolares de uma escola infantil pública. Rev Bras Obesidade, Nutrição e Emagrecimento 2015; 9(52):127-34.

31. Avena N, Rada P, Hoebel B. Evidence for sugar addiction: behavioral and neurochemical effects of intermitente excessive sugar intake. Neurosci Biobehav Rev 2008; 32(1):20-30.

32. Rosa M, Slavutzky S, Pechansky F, Keller F. Processo de desenvolvimento de um questionário para avaliação de abuso e dependência de açúcar. Cad Saúde Pública 2008; 24(8):1869-76.

33. Macêdo FS, Araújo MFM, Marinho BPN, Limas CA, Freitas DWR, Damasceno CMM. Fatores de risco para diabetes mellitus tipo 2 em crianças. Rev Lat Am Enfermagem 2014; 18(5):936-42.

\section{Endereço para correspondência:}

Juliana Figueiredo dal Gallo Faria

Rua Carlos Von Koseritz, 1415

90540-031 Porto Alegre, RS

Telefone: (51) 93089332

E-mail: ju.ffaria@hotmail.com

Recebido: 11 / 11 / 15. Aceito: 23 / 05 / 16. 